\title{
(Research Article) \\ Implementation of EV Battery Charging by Wireless Power Transfer
}

\author{
T. Kripalakshmi ${ }^{1}$, R. Ramaprabha ${ }^{2 *}$ \\ ${ }^{I}$ Post-graduation student, Department of Electrical \& Electronics Engineering, SSN college of Engineering, Kalavakkam, Tamil Nadu, INDIA \\ ${ }^{2 *}$ Associate Professor, Department of Electrical \& Electronics Engineering, SSN college of Engineering, Kalavakkam, Tamil Nadu, INDIA
}

\begin{abstract}
The transmission of electric power from a source point (front-end) to the load point (rear-end) destination offers to be a challenging one without any intermediate physical substance or either the annoying wires between them. As this originates from the experimentation of Testla in the year 1899 which is meant to be called as "World Wireless System", his experiment was done in the concept of microwave power generation which is most suitable for long distance and high frequency applications. Apart from the methods developed the promising wireless charging technology used is the ICPT by analyzing the design of charging coils, coupler, compensation network, power level enhancement and efficiency into major criteria. WPT is the technology that reduces the troubles in usages of wires and mainly long period charging of batteries. The objective of this paper aims in dynamically charging the online electric vehicle with the transmitter embedded inside the roads and the vehicle chassis as the receiver.
\end{abstract}

Keywords: Current magnification network, Loosely coupled coils, Dynamic charging, Magnetic resonance coupling.

\section{Introduction}

The wireless power transfer history began with the experiment of tesla coils and it has been categorized into two field techniques far-field technique and near-field technique. Electromagnetic waves was the transfer medium, the radiations emitted are hazardous to human beings. Magnetic resonance is the near field technique where an air-gap can used as a transfer medium. Magnetic coupled waves are similar to magnetic field induction of the transformer principle. Some of the technologies used are: Microwave Power Transfer (MPT), Inductive Power Transfer, and Inductively Coupled Power Transfer (ICPT) .Apart from the methods developed the promising wireless charging technology used is the ICPT by analyzing the design of charging coils, coupler, compensation network, power level enhancement and efficiency into major criteria. WPT is the technology that reduces the troubles in usages of wires and mainly long period charging of batteries [1]-[4].
Conductive charging method is replaced by the novel wireless power transfer (WPT) technology by maintaining a comparable power level and efficiency. The focus of this paper leads the way to dynamically power the moving vehicles on road with reduced battery pack and extending the charging distance. The charging troublesome in both the stationary and dynamic state of the electrical vehicle is discussed and addressed by some solutions presented below.

In Inductive Power Transfer (IPT) the coil structure is formed such that the two separated coupled coils or can be termed as a loosely coupled coils, they resonate at a frequency termed as resonant frequency hence this technology involves the resonant condition. The coils tend to operate at this resonance and, once tuned by either autotuning method or manual tuning method also to transfer power efficiently inspite of the air gap between the coils, efficiency, magnetic weak linkage and power transfer capability [5]-[9]. 
The prototype presented here is for small power range charging applications. The AC source comprises of additional power factor correction (PFC) circuit at the primary and secondary side. The front-end is provided here by the DC source and rear-end of the WPT circuit is provided with the battery regulatory system boost converter. The proposed WPT converter section is provided such that the induced primary and secondary voltage and its corresponding primary current are analyzed with the mutual inductor principle. Controlling power flow in IPT systems are available with two types of control the first one is either primary or secondary control or the other one is to provide the combination controller affecting both the primary and the secondary.

\section{Simulation of circuit and results}

The proposed WPT converter here is of capacitive DC load which can be assumed as a resistive load. The primary side is provided with high frequency power source which is responsible for the connection between the power grid and the magnetic link. It is usually composed by and rectifier followed by an inverter. The high frequency (HF) inverter $\mathrm{AC}$ output aids the inductive link to carry and induce current to the receiving coil. The HF inverter topology is a simple full-bridge circuit as shown in Figure 1. PWM technique was applied for the switches, the operation of the inverter is the same as the working of single phase full bridge inverter and the high frequency inverter implies not the switching frequency, the output of the inverter must provide as a high frequency inductive link to the primary transmitter coil.

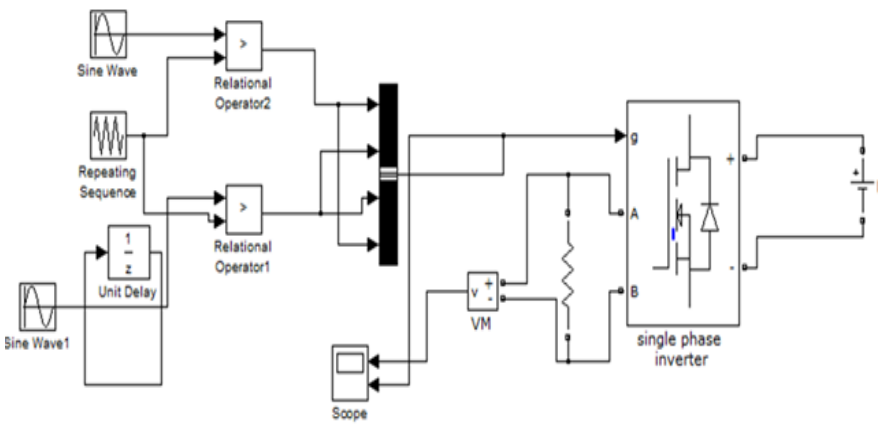

Figure 1. Full-Bridge HF inverter: Simulation circuit

The secondary side can be mentioned as the battery charger control circuit allows to control the charging process and to control the changing voltage to the levels accepted by each type of battery. It is also responsible by making the connection between the WPT system and the battery set to be charged which is on-board of the car. For a battery charging system a simple diode rectifier is used. The paper is proposed with the secondary side power converter with PFC [1] with power factor (pf) of 0.97 obtained from the simulation results (Figure 2).

*Corresponding Author: e-mail: ramaprabhar@ssn.edu.in ISSN 2320-7590

C 2018 Darshan Institute of Engg. \& Tech., All rights reserved

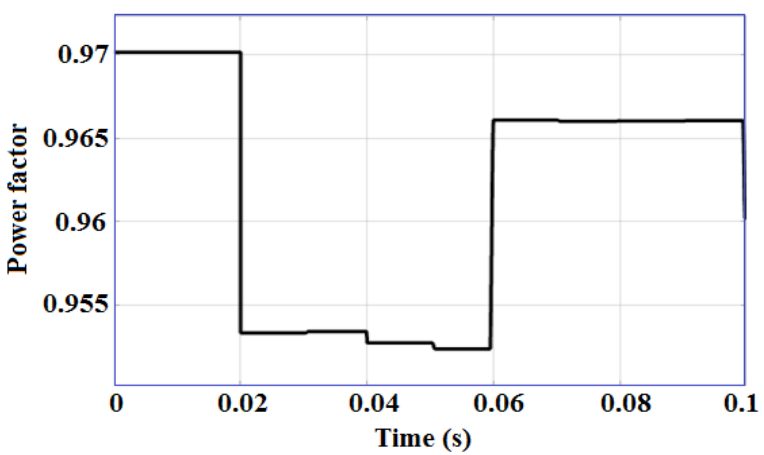

Figure 2. Secondary PFC circuit with pf value

As the value of power factor is approached to unity and the secondary side load is assumed here as a resistive load which is provided along with a battery charging regulatory circuit. The loosely coupled coils generally need to be compensated with a capacitor to obtain the current magnification; this network can be called as compensation network. For $k<0.3$, a compensation network is required on both sides for primary and secondary coils. The series-series compensation network is adopted in [1].The frequency response is simulated in PSpice (Figure 3) and the response is shown in Figure 4.

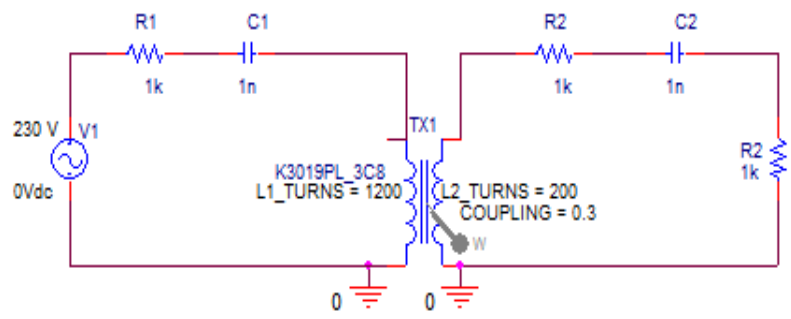

Figure 3. Compensation for $\mathrm{k}<0.3$

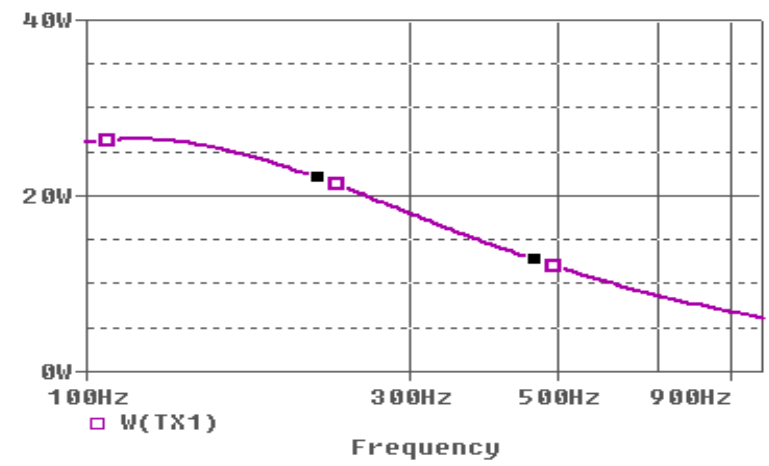

Figure 4. Frequency response characteristics

\section{Analysis of coils designed with coupling coefficient, $\mathrm{k}<0.5$}

The coils here is not wound on the same common magnetic core as they are separated at a distance from each other with $\mathrm{k}<0.5$, the leakage flux and the induced magnetic effect is reduced and become weaker. For weak flux linkage the compensation network is provided as seen in the previous 
section. The parameters need to be optimized for the coils to improve the efficiency between the coils are: .

- Quality factor $\left(Q_{L 1}, Q_{L 2}\right)$ for each coil

- Inductances of primary and secondary coils $\left(\mathrm{L}_{1}, \mathrm{~L} 2\right)$

- Tuning capacitance of primary side and secondary side $(\mathrm{C} 1, \mathrm{C} 2)$

- coupling coefficient $(\mathrm{k})$

- resonant frequency response $\left(f_{0}\right)$

The circuit for the optimization shown in Figure 5 and the equations are given through (1) to (5).

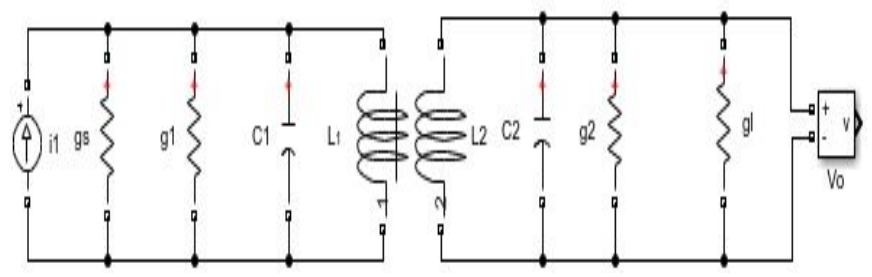

Figure 5. Circuit for optimization of parameters of the coils

$$
\begin{gathered}
Q_{L 1}=\frac{\omega_{0} C_{1}}{g_{t 1}}=\frac{1}{g_{t 1} \omega_{0 L_{1}}} \\
Q_{L 2}=\frac{\omega_{0} C_{2}}{g_{t 2}}=\frac{1}{g_{t 2} \omega_{0 L_{2}}} \\
Q_{L}=\sqrt{Q_{L 1} Q_{L 2}} \\
\mathrm{~K}=\sqrt[k]{Q_{L 1} Q_{L 2}}=\sqrt[k]{Q_{L}} \\
\omega_{0}=2 \pi f_{0}=\frac{1}{\sqrt{L_{1} C_{1}}}=\frac{1}{L_{2} C_{2}},
\end{gathered}
$$

where $\omega_{o}-$ resonant frequency

The coils have been simulated based on the critical parameters and from the previous analysis made with the help of coding in MATLAB including the distance between the coils and 3-D design of the coils is done in MAXWELL as in [1].

\section{Proposed Hardware Prototype}

Hardware prototype of WPT configuration was realized in Hylam sheet with all the components drilled with the terminal connections (banana terminals). Single phase inverter supply was given as a DC directly from the regulated power supply (RPS) $12 \mathrm{~V}$ and the pulses to the inverter were done using programming in ARDUINO controller interfaced with along with optocoupler; it isolates low voltage gate circuit from high voltage power circuit. To use a photo coupler to drive the high side of a bridge circuit, a floating power supply is required as shown in Figure 6.

The existing electric vehicle (EV) WPT designs are either too complex, uneconomical to implement with the higher frequency resonance operation hence for the small prototype the frequency operation is chosen in $\mathrm{kHz}$ operation. As per the simulation results, the prototype was done (Figure 7).

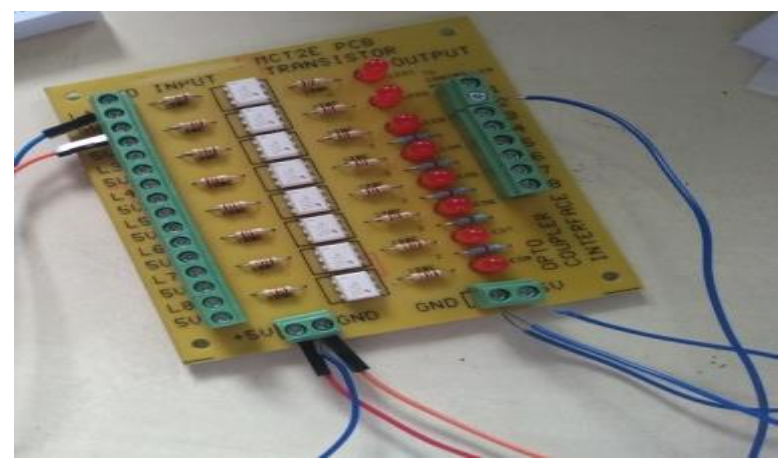

Figure 6. Optocoupler MCT2E Circuit

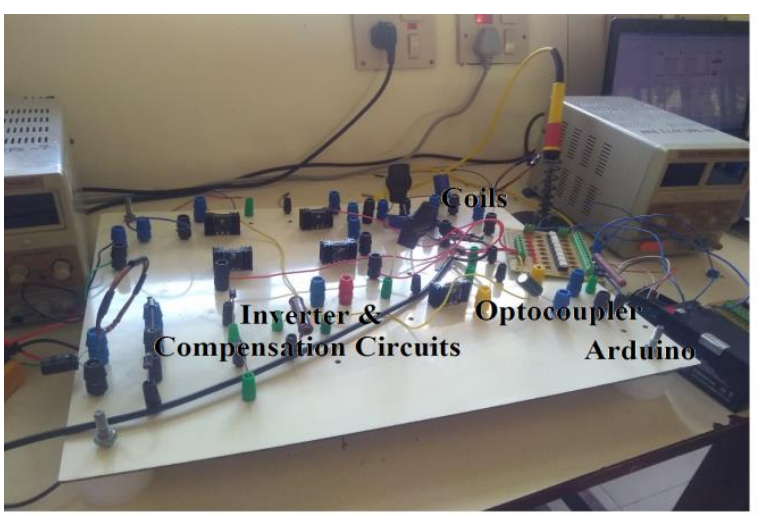

Figure 7. Hardware Prototype of Typical WPT Circuit

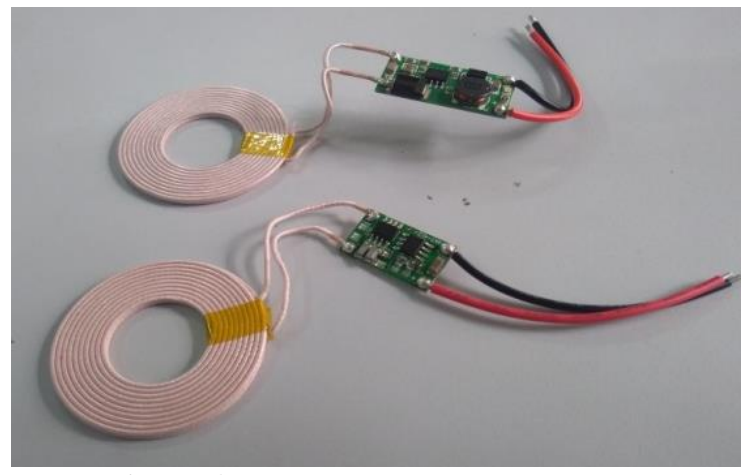

Figure 8. Litz wire: loosely coupled coils

The spiral shaped coils can transmit power more efficiently than the other geometry of the coils used in the literature. The number of turns and based on the optimization parameters as discussed in the previous topic the coil can be designed. 
Litz wire is used to reduce the skin proximity effect and the coils are provided along with the transmitter and receiver chip as we require a driver circuit to implement the coupled coils. The coils are shown in Figure 8. The primary side switched between the full bridge single phase inverter was done with the switching frequency and the PWM signals (Figure 9) and the high frequency output single phase voltage is with $20 \mathrm{kHz}$ frequency range is implemented and shown in Figure 10.

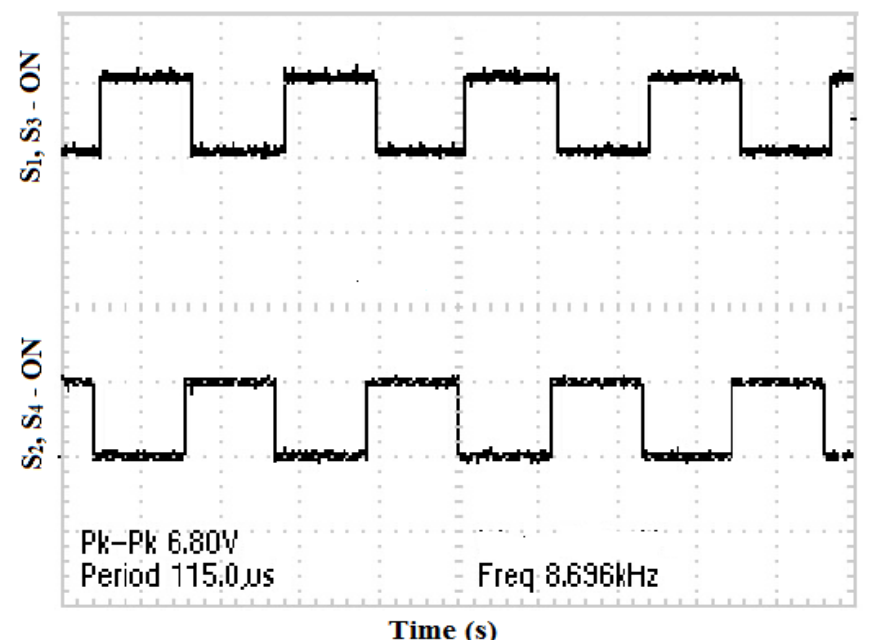

Figure 9. Switching pulses of high frequency inverter

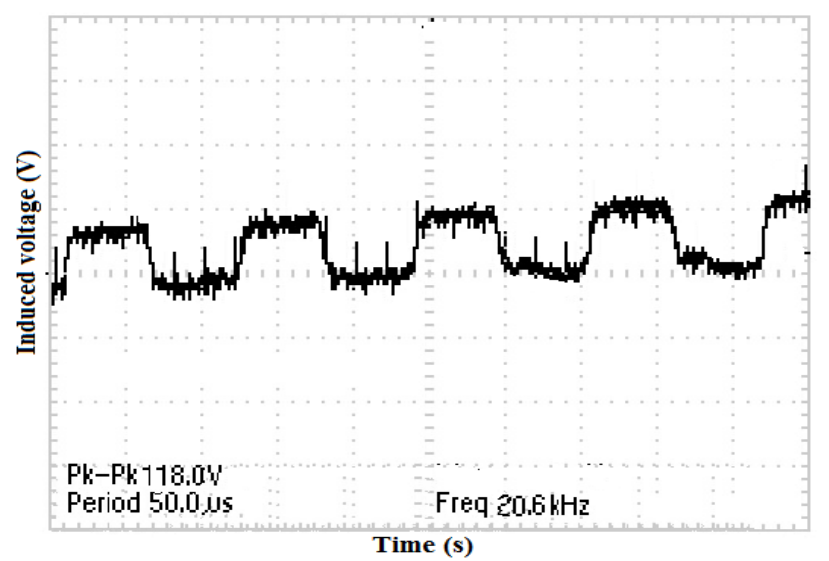

Figure 10. Output of single phase inverter high frequency signal

\section{Conclusion}

The wireless power transfer system for EV charging both primary control and secondary control can be enhanced and the loosely coupled coils challenges can be rectified with respect to increasing the distance and misalignment between the coils. Higher frequency resonating coils can be implemented with increasing beyond $20 \mathrm{kHz}$ that is done in this work. Power transfer and efficiency can be improved with complex and efficient coil design with a reasonably larger air gap. To maintain a normal operation of power transfer dual control has been adopted in this paper. The implementation was found to be contingent upon the leakage flux compensation topology, charging distance and proper alignment. The detailed of spiral coils has been presented in [1] by the authors. A trade-off between distance, charging pad size and efficiency is necessary for EV charging application.

\section{Acknowledgement}

I wish to thank the management of SSN College of Engineering, Chennai for providing internal funding and all the facilities in this project to carry out the work.

\section{References}

1. T. Kripalakshmi and R. Ramaprabha, Effect of Coupled Inductor on Wireless Power Transmission for Electric Vehicle Charging, 4th International Conference on Electrical Energy Systems (ICEES), pp. 328-332, Feb 2018.

2. Z. Wang, X. Wei and H. Dai, Principle Elaboration and System Structure Validation of Wireless Power Transfer via Strongly Coupled Magnetic Resonances, IEEE Vehicle Power and Propulsion Conference (VPPC), pp. 1-6,2013.

3. S. Li and C. C. Mi, Wireless Power Transfer for Electric Vehicle Applications, IEEE J. Emerg. Sel. Top. Power Electron,pp. 4-17, Mar. 2015.

4. M. Debbou and F. Colet, Inductive wireless power transfer for electric vehicle dynamic charging, IEEE PELS Workshop on Emerging Technologies Wireless Power Transfer, pp. 118-122, 2016.

5. A. I. Maswood and F. Liu, Unity power factor rectifier-inverter structure operating under unbalanced supply and variable dc bus voltage, IET Power Electronics, Vol.7 ,no.8 pp. 899-907, September 2011.

6. J. P. W. Chow, N. Chen, H. S. H. Chung and L. L. H. Chan, An Investigation Into the Use of Orthogonal Winding in Loosely Coupled Link for Improving Power Transfer Efficiency Under Coil Misalignment, IEEE Transactions on Power Electronics, vol. 30, no. 10, pp. 5632-5649, Oct. 2015.

7. G. A. J. Elliott, G. A. Covic, D. Kacprzak and J. T. Boys, A New Concept: Asymmetrical Pick-Ups for Inductively Coupled Power Transfer Monorail Systems in IEEE Transactions on Magnetics, vol. 42, no. 10, pp. 3389-3391, Oct. 2006.

8. S. Y. Choi, B. W. Gu, S. Y. Jeong and C. T. Rim, Advances in Wireless Power Transfer Systems for Roadway-Powered Electric Vehicles, IEEE Journal of Emerging and Selected Topics in Power Electronics, vol. 3, no. 1, pp. 18-36, March 2015. 
9. D. -. H. Tran, V. -. B. Vu, V.L. Pham ,Design and implementation of high efficiency Wireless Power Transfer system for on-board charger of Electric Vehicle," IEEE 8th International Power Electronics and Motion Control Conference (IPEMC-ECCE Asia), pp. 2466-2469,2016.

10. www.mathworks.com

\section{Biographical notes}

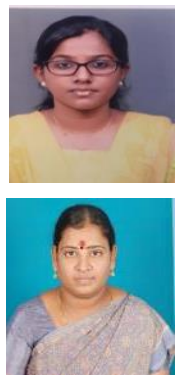

Ms.T.Kripalakshmi completed her post-graduation in engineering (M.E. Power Electronics and drives) in Electrical and Electronics department in SSN College of engineering. She has published one paper in IEEE conference.

Dr. R. Ramaprabha has received her B.E. in Electronics \& Communication Engineering from Periyar Maniammai College of Engineering Technology, Thanjavur in 1997 and M.E in Power Electrconics \& Drives from Shanmugha College of Engineering, Thanjavur in 2000. She completed her PhD from Anna University in 2011. She has been working in the teaching field for about 18 Years. She has published 32 papers in National conferences, 123 papers in referred International conferences and 93 papers in international journal in the area of Solar photovoltaic and Power electronics \& drives. She authored a text book in the area of power electronics for renewable energy systems. She obtained funded projects in her area of research. She is a life member in ISTE and senior member in IEEE 